\title{
Multimodal physical exercise and functional rehabilitation program in oncological patients with asthenia. study protocol
}

Eduardo José Fernández-Rodríguez 1,2,3, Jesús González-Sánchez ${ }^{1,3 *}$, Ana Silvia Puente-González ${ }^{1,3}$, José Ignacio Recio-Rodríguez ${ }^{1,3}$, Celia Sánchez-Gómez ${ }^{3,4}$, Roberto Méndez-Sánchez ${ }^{1,3}$, Juan Jesús Cruz-Hernández ${ }^{2,3,5}$ and María Isabel Rihuete-Galve 1,2,3 $^{-1,3}$

\begin{abstract}
Background: The increase in the survival of oncology patients include multiple side effects as cancer-related asthenia and dyspnea, which represents a serious health problem. An implementation of the conventional clinical practice, developed through multimodal physical exercise and functional rehabilitation program intervention, may be useful in controlling dyspnoea. This study aims to evaluate the effects of a multimodal exercise and functional rehabilitation program on fatigue, pain, functional capacity, and quality of life in cancer patients with cancer-related asthenia.

Methods: This is a protocol for an experimental, prospective, randomised study using a parallel, fixed assignment scheme, with an experimental group and a control group in patients from the oncology hospitalisation unit at the Salamanca University Hospital Complex in Spain, using consecutive sampling to select 50 participants with oncological asthenia who are hospitalised at the time of inclusion. After the baseline evaluation, the participants will be randomised into two groups. Both groups will receive standard clinical practice care and the normal health education program at discharge, but in addition, the participants assigned to the experimental group will also complete a multimodal exercise and functional rehabilitation program lasting one month. The primary outcomes will be basic activities of daily living (Barthel Index) and degree of asthenia (FACT-An scale). Additionally, physical performance will be evaluated with the Short Physical Performance Battery (SPPB), as will the attention and executive functions (Trail-Making Test), fear/avoidance of movement (TAMPA scale), pain (VAS scale), and body composition (waist, hip, brachial, thigh, wrist, and ankle circumferences).

Discussion: The results of this study may be translated to clinical practice, incorporating a specific autonomy recovery programme into comprehensive rehabilitation programmes of care for cancer patients with asthenia. The current study addresses to improve the conventional clinical practice by proposing a multimodal physical exercise and functional rehabilitation program intervention, which will be implemented by an interdisciplinary team, to try to improve the autonomy of cancer patients with cancer-related asthenia.
\end{abstract}

\footnotetext{
* Correspondence: jesusgonzsan@usal.es

${ }^{1}$ Department of Nursing and Physiotherapy, University of Salamanca,

Salamanca, Spain

${ }^{3}$ Institute of Biomedical Research of Salamanca (IBSAL), Salamanca, Spain

Full list of author information is available at the end of the article
}

\section{$\triangle B M C$}

(c) The Author(s). 2021 Open Access This article is licensed under a Creative Commons Attribution 4.0 International License, which permits use, sharing, adaptation, distribution and reproduction in any medium or format, as long as you give appropriate credit to the original author(s) and the source, provide a link to the Creative Commons licence, and indicate if changes were made. The images or other third party material in this article are included in the article's Creative Commons licence, unless indicated otherwise in a credit line to the material. If material is not included in the article's Creative Commons licence and your intended use is not permitted by statutory regulation or exceeds the permitted use, you will need to obtain permission directly from the copyright holder. To view a copy of this licence, visit http://creativecommons.org/licenses/by/4.0/ The Creative Commons Public Domain Dedication waiver (http://creativecommons.org/publicdomain/zero/1.0/) applies to the data made available in this article, unless otherwise stated in a credit line to the data. 
Trial registration: ClinicalTrials.gov; ID: NCT04761289. (February 18, 2021). https://clinicaltrials.gov/ct2/show/ NCT04761289.

Keywords: Nursing, cancer, cancer related fatigue, Physical exercise, Functional rehabilitation

\section{Background}

Nowadays, we observe an exponential increase in the five-year survival of patients with cancer, this is due to the improvement in preventive strategies as well as the greater knowledge about the oncologic pathology. Related to this higher life expectancy in cancer patients, an increase in the side effects of the treatments administered to surviving patients has also been observed [1]. These are mainly due to the increase in the lines of treatment employed. This increase produces a deterioration in the functionality and in the quality of life of the patients [2]. Some of these side effects include cancerrelated asthenia, depression and dyspnoea [3]. Cancerrelated asthenia is the most common symptom associated with cancer and its treatment, and it strongly impacts patient quality-of-life parameters [4]. However, this problem is still generally underestimated by many professionals in the field of oncology [5].

Patients with tumour asthenia often experience fatigue, which makes their daily lives difficult. In addition, the presence of fatigue is usually associated with alterations in body composition such as loss of muscle mass or an increase in visceral fat [6].

Among the intervention measures currently available to address this problem, the National Comprehensive Cancer Network (NCCN) expert panel considers educational measures, controlled therapeutic physical exercise, and energy conservation techniques as fundamental, always when undertaken within a complete functional rehabilitation program [7]. There is scientific evidence that asthenia can be better controlled after the implementation of non-pharmacological measures, especially controlled physical exercise [8, 9]. In addition, physical exercise has also been shown to mitigate the afore mentioned alterations in body composition that usually occur in cancer patients [10,11]. Indeed, Fernández-Lao et al. showed that patients with breast cancer who performed a multimodal physical exercise program achieved a reduction in body fat and an increase in lean body mass [12].

The available scientific literature indicates that the efficacy of these physical exercise interventions to address cancer-related fatigue is increased when a psychological component is added, especially when a interdisciplinary (oncology, nursing, physiotherapy, occupational therapy, and medicine) biopsychosocial approach is taken [13, 14]. Thus, different aspects related to loss-of-function, pain, and fatigue associated with fear-avoidance disorders should also be explored and evaluated by testing the degree of kinesiophobia in patients with cancer [15]. The cognitive-behavioural model of fear of movement notes that patients with chronic pain or fatigue syndrome tend to avoid physical activity because they believe that this activity causes or exacerbates these symptoms; avoidance behaviour leads to even greater fear and symptoms, producing more pain or fatigue [1517].

These factors can be extended to patients with cancerrelated asthenia, which is why physical activity is important to help them avoid loss-of-function and reduced physical capacity [15]. Selection of the optimal intervention environment is based on clinical complexity and the patient's ability to self-manage their situation. Several previous studies, including a meta-analysis of 14 randomised controlled clinical trials in breast cancer survivors with an intervention supervised by phone or email [18], have shown that supervised physical exercise interventions outside the healthcare provision environment, both in the community and at home, have had good results.

However, despite the potential benefits of such interventions, there is little evidence available on the possible effects that multimodal physical exercise and functional rehabilitation programs may have on patients with cancer-related asthenia outside healthcare settings. Thus, this present clinical trial was designed to address this research gap and to create empirical evidence regarding the effect of an interdisciplinary multimodal physical exercise and functional rehabilitation program intervention applied at the time of hospital discharge in patients with cancer-related asthenia.

From the above, we are proposing as an intervention a multimodal physical exercise and functional rehabilitation programme, implemented by an interdisciplinary team established by nurses, occupational therapists, physiotherapists, and specialist doctors, will improve the autonomy of patients with cancer-related asthenia. It would be a reeducation in the completion of the activities of daily living, so as to foment the recovery of personal autonomy. The findings will provide important insight into the development of an effective care model with a comprehensive rehabilitation programme that includes a specific autonomy recovery programme in oncological patients with asthenia. Taking into account the results obtained in our clinical practice in the Oncology Hospitalisation Unit at the University Hospital Complex of Salamanca (CAUSA), we consider important the 
implementation of specific clinical care protocols for this type of cancer patients, and its study through clinical trials.

\section{Methods/design}

\section{Design and aims}

This experimental, prospective, randomized, parallelcontrolled clinical trial, with two arms of fixed assignment with an experimental and a control group, will be conducted during a year in the Oncology Hospitalisation Unit at the CAUSA. We will use consecutive sampling to select the participants with oncological asthenia who are hospitalised at the time of inclusion.

This is one of the clinical trials that are starting in our Unit, with an interdisciplinary care team, and similar design, patients and procedures with others, such us the recent study protocol published [19].

According to the background, the primary aim of this study is to assess the effects of a multimodal physical exercise and functional rehabilitation program on fatigue, pain, functional capacity, and quality of life in cancer patients with cancer-related asthenia. The objectives are focused to compare the effects produced by the implementation of a multimodal physical exercise and functional rehabilitation program, with an isolated intervention using standard clinical practice treatment.

\section{Sample/participants}

Participants: The patients will be recruited from the Oncology Hospitalisation Unit at the CAUSA when they will be hospitalised. The Unit's investigators will invite patients to participate in the study by explaining the details of the clinical trial, and they can then be included once they have given their verbal and written consent, and meet the following selection criteria: (A) Inclusion criteria: Participants must have a pathological diagnosis of an oncological disease, be over 18 years of age, be hospitalised at the time of recruitment in the Oncology Hospitalisation Unit at the CAUSA, having a score of 15-55 points on the Barthel Index (BI), having a score of 4 or more on the visual-analogue scale (VAS) for cancer-related asthenia and have signed an informed consent form indicating voluntary agreement to participate in the study. (B) Exclusion criteria: not having an adequate cognitive state to be able to comprehend and follow the orders provided (fewer than 23 points on the Mini Mental State Examination, MMSE), and having a haemoglobin level lower than $10 \mathrm{~g} / \mathrm{dL}$. (C) Withdrawal criteria: disease progression which brings the patient to a terminal situation or to death, and non-completion of the follow-up and final assessments.

Assignment, randomisation and blinding: After recruitment, individuals will be assigned by a randomisation process to one of two study groups: Experimental Group or Control Group. Detailed in Fig. 1.

We will employ a simple randomisation process that consists of generating a table of random numbers of the same size as the estimated study sample with Microsoft Excel 2020 to assign subjects to each of the study groups. For even numbers, the subject will be assigned to the Experimental Group, and for odd numbers, the subject will be assigned to the Control Group. Randomisation, recruitment and assignment of subjects to each group will be performed by research staff not involved in the evaluations or interventions in each group, which will avoid potential bias in the study.

The participating subjects will not be blinded, due to the specific characteristics of the intervention in each group. However, the researchers responsible for the study measurements and statistical analysis will be blinded, with the intention of avoiding any contamination between groups, thus increasing the rigour of the study process.

Sample size: The sample size has been estimated based on the potential modification of one of the main outcome of the study-the score on the Barthel Index. For this calculation, we have considered the results obtained in a similar, where a modification of 17 points in the BI score was obtained, as a difference attributable to the intervention, considering this as the difference between the modification of the experimental group and the control group [20]. With these premises, accepting an alpha risk of 0.05 and a beta risk of 0.2 in a bilateral contrast, with 50 individuals ( 25 subjects in the experimental group and 25 in the control group, the statistical power was $90 \%$ to recognize as statistically significant a change of 17 points between both groups in the BI score. The sample size was estimated using the programme EPIDAT 4.2.

\section{Procedures and data collection Evaluations and study plan}

Participants will be assessed at three points during the study: Firstly, the baseline assessment at the beginning, the second assessment after seven days, and a third final assessment, before the patients are discharged from hospital. The baseline assessment will be performed after recruitment and before randomisation and assignment of subjects to the corresponding group. This initial assessment includes the recording of the independent variables and the primary and secondary outcome variables of the study. Then, after randomisation, the corresponding intervention will begin to be applied to each group. Subsequently, the other further assessments will be carried out, one after seven days, considered as the follow-up assessment, and another at hospital discharge, considered as the final assessment. This can be seen in more detail in Table 1. 


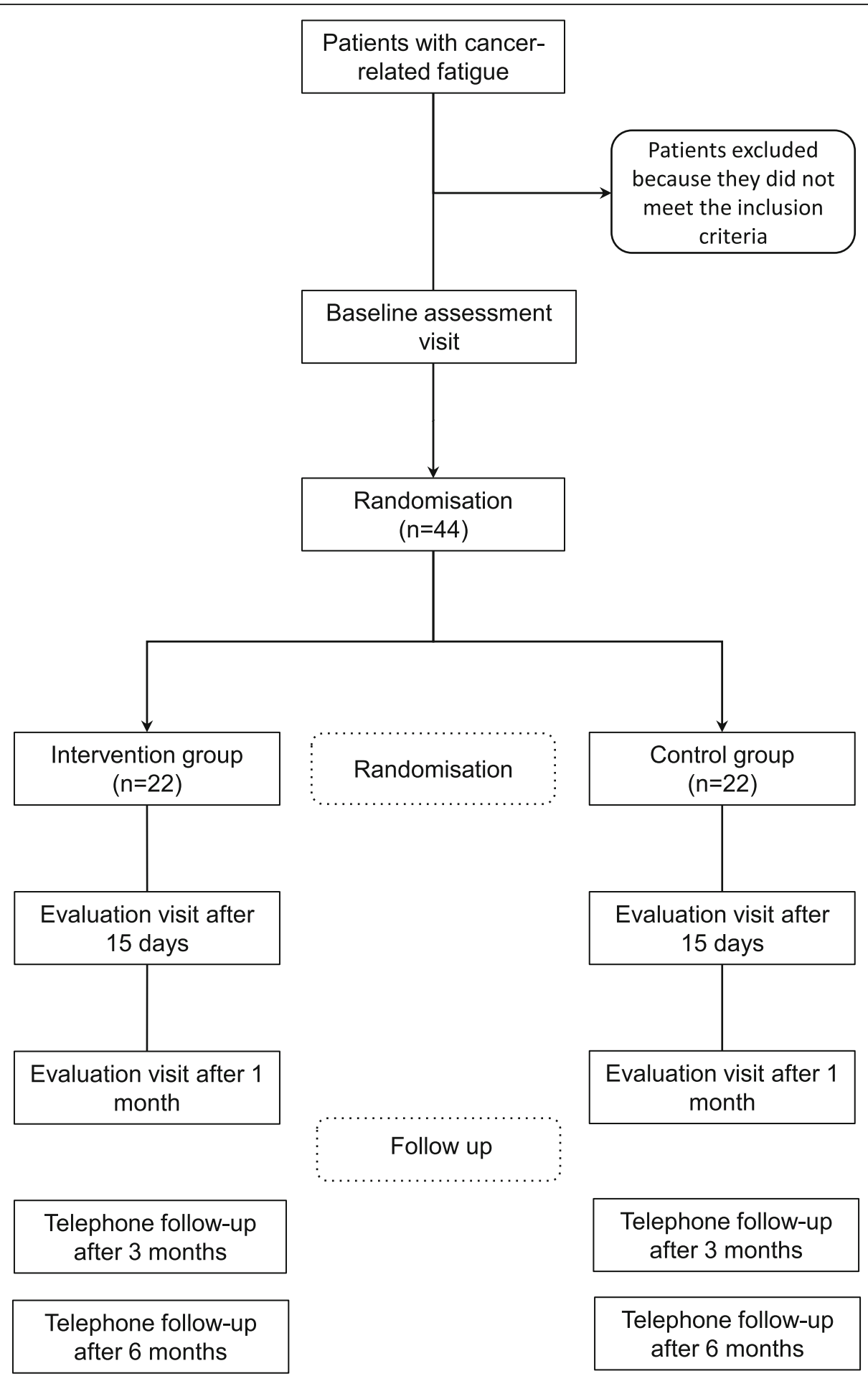

Fig. 1 Study design. The enrolled participants will be randomly assigned to one of the study groups and will be assessed at three pre-specified time $p$

\section{Description of the variables}

The main variable will be the degree of dependence in performing the activities of daily living (ADL), as measured using the Barthel Index. The secondary variables will be cancer-related asthenia, attention and cognitive functions, health-related quality of life (HRQoL), pain, functional capacity, fear/avoidance of movement using the Tampa Scale for Kinesiophobia associated with 
Table 1 Timeline and dimensions assessed at the time points

\begin{tabular}{|c|c|c|c|c|c|c|c|c|}
\hline \multirow[b]{3}{*}{ TIMEPOINT } & \multicolumn{8}{|c|}{ STUDY PERIOD } \\
\hline & \multirow{2}{*}{$\begin{array}{l}\text { Enrolment } \\
\text { Month 1-2 }\end{array}$} & \multirow{2}{*}{$\begin{array}{l}\text { Allocation } \\
\text { Month 2-3 }\end{array}$} & \multicolumn{2}{|c|}{ Post-allocation } & \multirow[b]{2}{*}{ M6 } & \multirow[b]{2}{*}{ M7 } & \multirow[b]{2}{*}{ M8 } & \multirow{2}{*}{$\begin{array}{l}\text { Close-out } \\
\text { M9-M12 }\end{array}$} \\
\hline & & & M4 & M5 & & & & \\
\hline \multicolumn{9}{|l|}{ ENROLMENT: } \\
\hline Eligibility screening & $x$ & & & & & & & \\
\hline Database preparation & $x$ & & & & & & & \\
\hline Informed consent & & $x$ & & & & & & \\
\hline Allocation & & $x$ & & & & & & \\
\hline \multicolumn{9}{|l|}{ INTERVENTIONS: } \\
\hline \multicolumn{9}{|l|}{ [Experimental Intervention] } \\
\hline \multicolumn{9}{|l|}{ [Control Intervention] } \\
\hline \multicolumn{9}{|l|}{ ASSESSMENTS: } \\
\hline [BASELINE ASSESSMENT] & & $x$ & & & & & & \\
\hline [FOLLOW-UP ASSESSMENT] & & & & & & & & $x$ \\
\hline [FINAL ASSESSMENT] & & & & & & & & $x$ \\
\hline DISSEMINATION OF RESULTS: & & & & & & & & $x$ \\
\hline
\end{tabular}

Fatigue (TSK-F), and body composition. We will also record the following intervening variables from the patient clinical history: pathological diagnosis, number of treatment lines, and other sociodemographic and anthropometric data.

\section{Tools employed in the evaluation of the variables}

1. "Barthel Index, (BI)" [21]: to evaluate dependence. Measures physical disability with proven validity and reliability. It is easy to apply and to interpret. Useful for evaluating the patient's functional independence in the activities of daily living (ADL). Scored from 0 to 100 , it quantifies the individual's degree of dependence.

2. "4th version of the Functional Evaluation of Cancer Therapy-Anaemia (FACT-An)" [22]: to evaluate cancer-related asthenia and the functional capacity. The FACT-An scale comprises the FACT-G fatigue subscale, plus seven non-fatigue items related to anaemia in cancer patients. In this study, we will use the version of FACT-G for cancer-related fatigue.

3. “Trail-Making Test (TMT)" [23]: to evaluate attention and executive functions. Useful for to assess attention, flexibility of thinking, and visuospatial ability. The global TMT comprises two parts: the TMT A measures attention, and the TMT B assesses processing speed and executive functions.

4. "EuroQol 5-D Questionnaire (EQ-5D)" [24]: to assess the health-related quality of life (HRQoL). It has been adapted and validated for use in the Spanish population.
5. “A Visual Analogue Scale (VAS)" [25, 26], to evaluate the pain. This will be assessed using the VAS as the most widely used pain assessment scale pain intensity is represented on a $10 \mathrm{~cm}$ line where 0 indicates the absence of pain and 10 represents the worst pain imaginable. The patient indicates the intensity of their pain; values less than 4 mean mild or mild-moderate pain; 4-6 indicate moderatesevere pain; and greater than 6 implies very intense pain.

6. "Short Physical Performance Battery (SPPB)" [27]: to evaluate Functional capacity. This test has been validated in the Spanish population for primary healthcare, and was specifically designed to predict disabilities, combines balance, gait speed, and the ability to get up from a chair and has been shown to predict adverse events, dependency, institutionalisation, and mortality.

7. The "Tampa Scale of Kinesiophobia-Fatigue (TSKF)" $[18,28]$, was developed to evaluate the fear of movement related to fatigue/pain. It has been proven effective in oncology patients and those with chronic fatigue syndrome. We will use the model with 11 items (TSK-F-11).

8. Evaluation of body composition: by measuring the waist, hip, brachial, thigh, wrist, and ankle circumferences. Height and weight will be measured using a portable stadiometer (Seca, 222), calculating the average of two recorded measurements.

An individual data collection sheet will be used for each patient and recorded in a database specifically designed for this study. 


\section{Interventions}

Conventional standard of care will be applied in both groups, explaining to each subject participating in the study the importance of compliance and adherence to the prescribed pharmacological treatment and the established guidelines personalised to their specific health needs. In the days prior to hospital discharge, they will also receive, as a part of the intervention, a health education programme with an informative talk and written guidelines, mainly focused at reinforcing and promoting an active and healthy lifestyle.

The difference of the intervention in the experimental group will be due to the fact that we will also implement the intervention with a multimodal programme of physical exercise and functional rehabilitation lasting one month. This will consist of:

1. A prescription for multimodal physical exercise. In the Oncology Hospitalisation Unit at the CAUSA, as in other studies already initiated [19], we will carry out the following design of a multimodal physical exercise programme. A supervised and structured program will be implemented in these patients for one month. This will consist of two short 15-20-minute sessions performed daily, one in the morning and one in the afternoon. The sessions will be structured according to the recommendations of the American College of Sports Medicine (ACSM) [29, 30], with an initial warm-up (2$3 \mathrm{~min})$, the main section comprising the principal physical exercises (8-12 min), and a final cool-down and relaxation phase $(5 \mathrm{~min})$.

Parts of a standard session: The warm-up will consist of exercises designed to promote joint mobility and muscle activation. The main section will combine aerobic and balance exercises with low-load strength exercises targeting upper and lower quadrant muscle groups. The dosage and load will always be adapted according to patient evaluations performed beforehand and will follow a progression in difficulty with the aim of achieving an intensity at 50-75\% of each patient's maximum heart rate or a perception of moderate effort on the Borg Scale. After 15 days of the program, the second evaluation will be carried out and the program may then be modified by adapting the load. Finally, the cool down will consist of gentle muscle stretching and relaxation exercises.

2. Activities of daily living re-education. Specific training designed to identify factors impeding the performance of the ADL will be conducted after the assessment and before the time of patient discharge from the hospital. This intervention will comprise three parts: (1) a direct ADL intervention carried out in situ in the hospital which will be generalisable to the patient's everyday environment; (2) teaching of energy saving techniques (ESTs) based on the simplification of activities; and (3) advice on sleep hygiene measures for sleep disorders, as outlined in the specific NCCN guidelines on cancerrelated asthenia [31].

3. Prescription of assistive products and environmental adaptations. Before the patient is discharged and after the baseline evaluation, we will evaluate if any support products that will favour their autonomy can be prescribed and at the time of hospital discharge and we will identify possible barriers in their home that could impair their autonomy.

\section{Work plan and visit structure}

Once the possible study candidates have been identified in the Oncology Hospitalisation Unit at the CAUSA, an interview with each individual will be organised to explain the purpose of the study to them and thereafter, invite them to sign the informed consent to their participation. Each participant will complete 3 planned evaluation visits. One at baseline, prior to randomisation, and two follow-up visits 15 days and 1 month after the first visit. The structure of all three 3 visits will be the same and each will last approximately one hour. In addition, there will be a telephone follow-up 3 and 6 months after the randomisation. The study variables will be evaluated during these visits, as specified in Table 1.

\section{Baseline visit}

This will be performed prior to patient discharge and ensuring compliance with the selection criteria. The evaluation will involve collection of all the study variables and completion of the questionnaires at baseline; the patient sociodemographic data, medical history, presence of comorbidities, and use of concomitant medications will also be recorded. At the end of this visit the patients will be randomly assigned to one of the two study groups. Participants included in the experimental group will then be explained the structure and organisation of the functional rehabilitation program during a specific visit planned for this purpose.

\section{Follow-up visits at 15 days and 1 month}

These visits will be identical to the baseline evaluation, except that the sociodemographic variables will only be collected at the baseline evaluation. The follow-up visits will be completed in the Clinical Teaching Assistance Unit in the Faculty of Psychology at the University of Salamanca, Spain.

\section{Data analysis}

The statistical analysis will be carried out on an intention-to-treat basis. The characteristics of the population will be presented as means and standard deviations for continuous variables and as a frequency distribution for qualitative variables. To assess 
comparability of the two study groups at the baseline, the means between both groups will be compared using chi-square tests for qualitative variables and Student $t$ tests for qualitative variables. The effect of the intervention on the study variables will be evaluated using a repeated measures ANOVA with two study factors: time * group. Subgroup analyses will be carried out considering certain variables and/or categories from the baseline evaluation such as age or the initial FACT scale score. For hypothesis testing, an alpha risk of 0.05 will be set as the limit for tests of statistical significance. We will use SPSS software (version 23.0; IBM Corp., Armonk, NY) for all the statistical analyses.

\section{Rigour}

This protocol study also follows the evidence-based recommendations of The SPIRIT 2013 Statement for the minimum content of a clinical trial protocol. And the design of the study also follows the evidence-based, minimum set of recommendations of the CONSORT 2010 Statement for conduct parallel-group randomized controlled trials (RCT), enabling readers to understand a trial's design, conduct, analysis and interpretation, and to assess the validity of its results.

Data availability: Upon completion of the study, the data gathered herein will be made available to those investigators who request it through FAIRsharing of the repositories of research data at https://www.re3data.org/.

\section{Discussion}

Cancer-related asthenia is considered the most common symptom associated with tumours and their treatment and is an essential factor in the functional deterioration of individuals with cancer [5]. There is growing interest in conducting clinical trials on the influence of physical exercise on the quality of life of cancer patients, although how these beneficial influences might affect the autonomy of patients in their daily lives remains to be addressed. However, these beneficial effects have been observed in daily clinical practice in patients with cancer-related asthenia in terms of physical improvements. Nonetheless, difficulties in generalising this physical improvement to occupational performance have also been noted [7-9].

As oncological clinicians, one of the biggest problems we face on a daily basis is that, although the acute patient symptoms will have been optimally resolved at the time of hospital discharge, there is still room for improvement in functional terms. With the planned follow-up described in this article, we intend to resolve this handicap by incorporating a prescription for specific rehabilitation methods for patients. We consider it essential to include ADL re-education techniques as part of the multimodal physical exercise rehabilitation methods [9].

Furthermore, in order to favour both an adequate intervention environment as well as self-management of situations by patients, our study proposes a supervised home-based intervention adapted to the specific situation of each patient with moderate cancer-related asthenia for implementation after their discharge from hospital. This meets the NCCN recommendations that better, more accessible physical exercise programs should be offered which patients are able to easily adhere to. In addition, they also suggest that the prescription of support products should be incorporated as these will contribute to achieving greater immediate functional improvement of individuals.

\section{Limitations}

This study will follow all the recommendations of the CONSORT guidelines, but due to the nature of the intervention itself, the participants will not be blinded to the intervention. However, the researchers responsible for carrying out the study measurements in each evaluation and for the statistical analysis will be blinded.

\section{Dissemination plan}

Dissemination of the study results will be carried out with the intention of ensuring maximum visibility. The results will be published in peer-reviewed open access scientific journals. There will be an initial publication of the primary results, with further publications of the secondary results planned. The results will also be presented at major national and international scientific conferences and seminars. Finally, the research team and the Oncology Hospitalisation Unit at the CAUSA will disseminate the results in social networks and other media.

\section{How potential changes in the study will be approached}

Significant modifications to the protocol (such as change in the tools of evaluation, modifications to the selection criteria or to the interventions) will be communicated immediately to the bioethics committee.

\section{Conclusions}

This study protocol aimed at assessing the influence of incorporating an interdisciplinary intervention, carried out by nurses, occupational therapists, physiotherapists, and specialist doctors in patients with cancer-related fatigue, with the aim of improving conventional clinical practice, and introducing a program of multimodal physical exercise and functional rehabilitation, which we consider essential in the follow-up of patients once they are discharged from hospital. 


\section{Abbreviations}

SPPB: Short Physical Performance Battery; NCCN: National Comprehensive Cancer Network; VAS: visual-analogue scale; MMSE: Mini-Mental State Examination; BI: Barthel Index; HRQoL: Health-related quality of life; TSKF: Tampa Scale for Kinesiophobia associated with Fatigue; FACTAn: Functional Evaluation of Cancer Therapy-Anaemia; TMT: Trail-Making Test; EQ-5D: EuroQol 5-D Questionnaire; ACSM: American College of Sports Medicine; ADL: Activities of daily living

\section{Acknowledgements}

The investigative team would like to express their appreciation to all of the patients who will take part in the study for their participation, as well as to all of our professional colleagues who will contribute in some way to the optimal development of this study.

\section{Authors' contributions}

EJFR, MIRG, and JGS are the principal investigators; they designed the study and are responsible for the trial design and study procedures. JJCH and CSG are responsible for recruitment and the patients' information. ASPG and RMS are responsible for the interventions and for the supervision of the study and the participants. JIRR and JGS are responsible for the statistical analysis. JJCH, JIRR, EJFR, RMS, CSG, and MIRG are responsible for the preparation of publications. All authors read and approved the final manuscript.

\section{Funding}

This study was funded by the Regional Health Management of Castile and León, Spain, with project file number 2181/A/2020, and has undergone peerreview by the funding body. The funding is external and not industry funded.

\section{Availability of data and materials}

Upon completion of the study, the datasets generated and/or analysed during the current study will be available to those investigators who request it through FAlRsharing of the repositories of research data at https://www. re3data.org/

\section{Declarations}

\section{Ethics approval and consent to participate}

The study was approved by the Clinical Research Ethics Committee of the Area of Health of Salamanca (ID: PI 202007547), having obtained the prior written informed consent of the study subjects and in conformance with the Helsinki Declaration. The participants will be informed of the objectives of the project and the risks and benefits of the interventions that will be carried out during the work. Participants will be fully informed, and we will guarantee that their data will be treated with the utmost confidentiality throughout the study and also after its completion. Consequently, data and information will not be shared with third parties. Researchers involved in the study will strictly adhere to professional confidentiality and the confidentiality of the participants will be guaranteed at all times in accordance with the laws on the protection of personal data and biomedical research presented in the provisions of Organic Law 3/2018 of December 5 on the Protection of Personal Data and the Guarantee of Digital Rights, Regulation (EU) 2016/679 of the European Parliament and Council of 27 April 2016 on data protection (GDPR), and under the conditions established by Law 14/2007 on biomedical research.

\section{Consent for publication}

Not applicable.

\section{Competing interests}

The authors declare that they have no competing interests.

\section{Author details}

'Department of Nursing and Physiotherapy, University of Salamanca, Salamanca, Spain. ${ }^{2}$ Medical Oncology Service, University Hospital Complex of Salamanca, Salamanca, Spain. ${ }^{3}$ Institute of Biomedical Research of Salamanca (IBSAL), Salamanca, Spain. ${ }^{4}$ Department of Developmental and Educational Psychology, University of Salamanca, Salamanca, Spain. ${ }^{5}$ Department of Medicine, University of Salamanca, Salamanca, Spain.
Received: 7 August 2021 Accepted: 12 October 2021

Published online: 22 October 2021

\section{References}

1. Bray F, Ferlay J, Soerjomataram I, Siegel RL, Torre LA, Jemal A. Global cancer statistics 2018: GLOBOCAN estimates of incidence and mortality worldwide for 36 cancers in 185 countries. CA Cancer J Clin. 2018;68:394-424.

2. Siegel RL, Miller KD, Jemal A. Cancer statistics, 2020. CA Cancer J Clin. 2020; 70:7-30.

3. Koelwyn GJ, Jones LW, Hornsby W, Eves ND. Exercise therapy in the management of dyspnea in patients with cancer. Curr Opin Support Palliat Care. 2012;6:129-37.

4. Hofman M, Ryan JL, Fiqueroa-Moseley CD, Jean-Pierre P, Morrow GR. Cancer-related fatigue: the scale of the problem. Oncologist. 2007;12(Suppl 1):4-10.

5. Henry DH, Viswanathan HN, Elkin EP, Traina S, Wade S, Cella D. Symptoms and treatment burden associated with cancer treatment: results from a cross-sectional national survey in the U.S. Support care cancer Off. J Multinatl Assoc Support Care Cancer. 2008;16:791-801.

6. Neefjes ECW, van den Hurk RM, Blauwhoff-Buskermolen S, van der Vorst MJDL, Becker-Commissaris A, de van der Schueren MAE, et al. Muscle mass as a target to reduce fatigue in patients with advanced cancer. J Cachexia Sarcopenia Muscle. 2017;8:623-9.

7. Berger AM, Mooney K. Dissemination and Implementation of Guidelines for Cancer-Related Fatigue. J Natl Compr Canc Netw. 2016;14:1336-8.

8. Wu C, Zheng Y, Duan Y, Lai X, Cui S, Xu N, et al. Nonpharmacological Interventions for Cancer-Related Fatigue: A Systematic Review and Bayesian Network Meta-Analysis. Worldviews evidence-based Nurs. 2019;16:102-10.

9. Barsevick AM, Dudley W, Beck S, Sweeney C, Whitmer K, Nail L. A randomized clinical trial of energy conservation for patients with cancerrelated fatigue. Cancer. 2004;100:1302-10.

10. Keilani M, Hasenoehrl T, Baumann L, Ristl R, Schwarz M, Marhold M, et al. Effects of resistance exercise in prostate cancer patients: a meta-analysis. Support care cancer Off J Multinatl Assoc Support Care Cancer. 2017;25: 2953-68.

11. Yen C-J, Hung C-H, Kao C-L, Tsai W-M, Chan S-H, Cheng H-C, et al. Multimodal exercise ameliorates exercise responses and body composition in head and neck cancer patients receiving chemotherapy. Support care cancer Off J Multinatl Assoc Support Care Cancer. 2019;27:4687-95.

12. Fernández-Lao C, Cantarero-Villanueva I, Ariza-Garcia A, Courtney C, Fernández-de-las-Peñas C, Arroyo-Morales M. Water versus land-based multimodal exercise program effects on body composition in breast cancer survivors: a controlled clinical trial. Support care cancer Off J Multinatl Assoc Support Care Cancer. 2013;21:521-30.

13. Schmitz KH, Campbell AM, Stuiver MM, Pinto BM, Schwartz AL, Morris GS, et al. Exercise is medicine in oncology: Engaging clinicians to help patients move through cancer. CA Cancer J Clin. 2019;69:468-84.

14. Campbell KL, Winters-Stone KM, Wiskemann J, May AM, Schwartz AL, Courneya KS, et al. Exercise Guidelines for Cancer Survivors: Consensus Statement from International Multidisciplinary Roundtable. Med Sci Sports Exerc. 2019;51:2375-90.

15. Bluethmann SM, Vernon SW, Gabriel KP, Murphy CC, Bartholomew LK. Taking the next step: a systematic review and meta-analysis of physical activity and behavior change interventions in recent post-treatment breast cancer survivors. Breast Cancer Res Treat. 2015;149:331-42.

16. Sheeran P, Abraham C, Jones K, Villegas ME, Avishai A, Symes YR, et al. Promoting physical activity among cancer survivors: Meta-analysis and meta-CART analysis of randomized controlled trials. Heal Psychol Off J Div Heal Psychol Am Psychol Assoc. 2019:38:467-82.

17. Turner RR, Steed L, Quirk H, Greasley RU, Saxton JM, Taylor SJ, et al. Interventions for promoting habitual exercise in people living with and beyond cancer. Cochrane database Syst Rev. 2018;9:CD010192.

18. Velthuis MJ, Van den Bussche E, May AM, Giijsen BCM, Nijs S, Vlaeyen JWS. Fear of movement in cancer survivors: validation of the modified Tampa scale of kinesiophobia-fatigue. Psychooncology. 2012;21:762-70.

19. Fernández-Rodríguez EJ, González-Sánchez J, Puente-González AS, RecioRodríguez Jl, Sánchez-Gómez C, Méndez-Sánchez R, Cruz-Hernández JJ, Rihuete-Galve MI. Specific autonomy recovery programme in a comprehensive rehabilitation on functionality and respiratory parameters in oncological patients with dyspnoea. Study protocol. BMC Nurs. 2021 Jul;5(1): 120. 20 (. 
20. Fernández Rodríguez EJ, Rihuete Galve MI, Cruz Hernández JJ. Impact of a comprehensive functional rehabilitation programme on the quality of life of the oncological patient with dyspnoea. Med Clin (Barc). 2021;157(1):10-6.

21. Mahoney Fl, Barthel DW. Functional Evaluation: The Barthel Index. Md State Med J. 1965 Feb;14:61-5.

22. Cella D. The Functional Assessment of Cancer Therapy-Anemia (FACT-An) Scale: a new tool for the assessment of outcomes in cancer anemia and fatigue. Semin Hematol. 1997;34(3 Suppl 2):13-9.

23. Reitan RM. Trail Making Test: Manual for administration and scoring. Reitan Neuropsychology Laboratory; 1992.

24. Badia X, Schiaffino A, Alonso J, Herdman M. Using the EuroQol 5-D in the Catalan general population: feasibility and construct validity. Qual life Res an Int J Qual life Asp Treat care Rehabil. 1998;7:311-22.

25. Ho K, Spence J, Murphy MF. Review of pain-measurement tools. Ann Emerg Med. 1996:27:427-32.

26. Collins SL, Moore RA, McQuay HJ. The visual analogue pain intensity scale: what is moderate pain in millimetres? Pain. 1997:72:95-7.

27. Martinez-Monje F, Cortés-Gálvez JM, Cartagena-Pérez Y, Alfonso-Cano C, Sánchez-López MI, Leal-Hernández M. Assessment with Short Physical Performance Battery Scale of Functional Ability in the Elderly over 70 years. Atención Fam. 2017;24:145-9.

28. Nijs J, Roussel N, Van Oosterwijck J, De Kooning M, Ickmans K, Struyf F, et al. Fear of movement and avoidance behaviour toward physical activity in chronic-fatigue syndrome and fibromyalgia: state of the art and implications for clinical practice. Clin Rheumatol. 2013;32:1121-9.

29. Schmitz KH, Courneya KS, Matthews C, Demark-Wahnefried W, Galvão DA, Pinto BM, et al. American College of Sports Medicine roundtable on exercise guidelines for cancer survivors. Med Sci Sports Exerc. 2010;42:140926.

30. Thompson PD, Arena R, Riebe D, Pescatello LS. ACSM's new preparticipation health screening recommendations from ACSM's guidelines for exercise testing and prescription, ninth edition. Curr Sports Med Rep. 2013;12:215-7.

31. Rihuete Galve MI, Fernández Rodríguez EJ. Effectiveness of educational program on quality of life of fatigue reported by the oncologic disease. Fundación Index; 2018. https://scielo.isciii.es/scielo.php?script=sci_a rttext\&pid=S1132-12962018000100002\&lng=es\&nrm=iso\&tlng=es.

\section{Publisher's Note}

Springer Nature remains neutral with regard to jurisdictional claims in published maps and institutional affiliations.

Ready to submit your research? Choose BMC and benefit from:

- fast, convenient online submission

- thorough peer review by experienced researchers in your field

- rapid publication on acceptance

- support for research data, including large and complex data types

- gold Open Access which fosters wider collaboration and increased citations

- maximum visibility for your research: over $100 \mathrm{M}$ website views per year

At $\mathrm{BMC}$, research is always in progress.

Learn more biomedcentral.com/submissions 